The objective of syndromic surveillance is to identify illness clusters early and to mobilise a rapid response, thereby reducing morbidity and mortality.

Objective To describe the system named Bonis, which uses Information and Communication Technology to prevent, warn, monitor and control the spread of febrile syndromes and influenza like illness (ILI) at the community level.

Methods Bonis has a kernel free software application; Asterisk, a phone center with VoIP service, PHP as Script language and Mysql as data base management. The system was implemented in a primary healthcare.

Results The developed system has the ability to record, classify, and prioritise automatically through the Interactive Voice Response the suspected cases. It has been programmed in a PHP language script AGI to improve the functionality of the Asterisk, in a way that during the user call to report a possible case, the system feeds a database, through the manager module based in a web application developed in PHP. The system, to which the users access from a mobile or fixed telephone, automatically receives the call with 9 questions on signs and symptoms. The system is working since May 2010 and records the phone calls correctly; follow-ups of these patients are performed by the teams within a period of $24 \mathrm{~h}$.

Conclusion The developed system is allowing the community to notify and register events that require surveillance, and the community health agents is prioritising visits to those homes from where fever cases are reported for sooner and more appropriate interventions.

\section{P1-105 INCIDENCE OF SEVERE ACUTE RESPIRATORY INFECTIONS (SARI) AND DEATHS ASSOCIATED WITH INFLUENZA}

doi:10.1136/jech.2011.142976c.98

\begin{abstract}
${ }^{1,2} \mathrm{~A}$ Cabello, ${ }^{*} \mathrm{M}$ V Horoch, ${ }^{3} \mathrm{~L}$ Bobadilla, ${ }^{3} \mathrm{C}$ Vazquez, ${ }^{1,2} \mathrm{M}$ Samudio, ${ }^{1}$ I Allende. ${ }^{1}$ Dirección General de Vigilancia de la Salud (DGVS), Asuncion, Paraguay; ${ }^{2}$ Instituto de Investigaciones en Ciencias de la Salud.UNA, Asuncion, Paraguay; ${ }^{3}$ Laboratorio Central de Salud Publica, Asuncion, Paraguay
\end{abstract}

Background Respiratory infections are one of the leading causes of morbidity and mortality worldwide: influenza is one of the predominant pathogens responsible. During 2010, Paraguay actively sought for $\mathrm{pH} 1 \mathrm{~N} 1$ case-patients to estimate the incidence of severe acute respiratory infections (SARI) and deaths associated with influenza.

Objective To determine the contribution of Influenza in patients hospitalised with clinical evidence of severe acute respiratory infections (SARI).

Methods During 1 January 2010-31 December 2010, hospital staff identified all case-patients who met the SARI definition of sudden onset fever, and cough or sore throat, with shortness of breath requiring hospitalisation. Physicians obtained nasal and pharyngeal swabs from case-patients and sent the samples to the Paraguay National Influenza Center for influenza testing by immunofluorescence and reverse transcriptase polymerase chain reaction.

Results Hospital staff identified 2145 SARI cases of which they tested $1581(74 \%)$ for influenza. Of these 284 cases (18\%) were positive for influenza. The most frequently subtypes identified viruses were Influenza A H3N2 71\% (202/284), influenza A H1N1 $14 \%(41 / 284)$, influenza B 14\% (41/284). We estimated that the rate of influenza-associated mortality was 2.8/100 000 persons-year (py). Conclusion Out findings suggest that influenza caused a significant burden and may warrant further investments in its control and prevention.

\section{P1-106 CAPTURE-RECAPTURE ANALYSIS OF ALL-CAUSE MORTALITY DATA IN BOHOL, PHILIPPINES}

doi:10.1136/jech.2011.142976c.99

${ }^{1} \mathrm{~K}$ Carter, ${ }^{*}{ }^{1} \mathrm{G}$ Williams, ${ }^{2} \mathrm{~V}$ Tallo, ${ }^{2} \mathrm{D}$ Sanvictores, ${ }^{2} \mathrm{H}$ Madera, ${ }^{1} \mathrm{I}$ Riley. ${ }^{1}$ School of Population Health, University of Queensland, Herston (Brisbane),
Queensland, Australia; ${ }^{2}$ Research Institute of Tropical Medicine, Manila, The Philippines

Introduction Despite the importance of mortality data, official reporting systems rarely capture every death. Completeness of death reporting and the subsequent effect on mortality estimates was examined in Bohol province in the Philippines using a system review and capture-recapture analysis.

Methods Records of deaths were collected from local civil registration offices, health centres and hospitals, and parish churches, and reconciled using a specific set of matching criteria. Two and three source capture-recapture analysis was conducted. For the two-source analysis civil registry and health data were combined due to dependence between these sources, and analysed against church data. Results Significant dependence between civil registration and health reporting systems was identified. There were 8075 unique deaths recorded in the study area between 2002 and 2007. Government records capture only $77 \%$ of deaths, while $5 \%-10 \%$ of deaths were not reported to any source. Average life expectancy (2002-2007) was estimated at 65.7 years and 73.0 years for males and females respectively, 4-5 years lower than estimated from civil registration data alone. Reporting patterns varied by age and municipality with childhood deaths more under-reported than adult deaths. Infant mortality was under-reported in civil registration data by $62 \%$.

Conclusion Deaths are under-reported in Bohol and uncorrected mortality measures would subsequently be misleading if used for health planning and evaluation purposes. These findings highlight the importance of ensuring official mortality estimates from the Philippines are derived from data that has been assessed for underreporting and corrected as necessary.

\section{P1-107 CONTEXTUAL CIRCUMSTANCES AND PATTERNS OF CHILDHOOD WEIGHT CHANGE}

doi:10.1136/jech.2011.142976c.100

${ }^{1} \mathrm{M}$ A Carter, ${ }^{*}{ }^{1} \mathrm{~L}$ Dubois, ${ }^{1,2} \mathrm{M}$ S Tremblay. ${ }^{1}$ University of Ottawa, Ottawa, Ontario, Canada; ${ }^{2}$ Healthy Active Living and Obesity Research, Children's Hospital of Eastern Ontario, Ottawa, Ontario, Canada

Introduction Worldwide, the prevalence of childhood obesity has not abated, indicating that prevention strategies, traditionally implemented at the individual- level, may not be effective. Conceptualising childhood obesity within multiple levels of influence, specifically within residential communities and over the lifecourse, is necessary to design effective prevention strategies that shift the distribution of risk downward.

Methods Participants of the Québec Longitudinal Study of Child Development $(n=1588)$ comprised the sample for analysis. Standardised BMI measurements from 4 to $10 \mathrm{y}$ of age and a semiparametric mixture modelling method were used to estimate developmental trajectories of weight change. The influence of the residential environment on weight trajectories was estimated after controlling for social and early life factors, such as SES and birthweight.

Results Four distinct weight trajectory groups were estimated: (1) Low- increasing (7.1\%), (2) Low/medium-increasing (35.2\%), (3) Medium/high- increasing (47.4\%), and 4) High-stable (10.3\%). Switching from urban to rural living decreased weights in Group 1, but increased weights in Group 4. In Group 2, changing from urban to medium density living increased weights. For Group 1, moving to a more cohesive neighbourhood increased weights, and moving to a more highly disordered neighbourhood decreased weights. Compared to the other three groups, Group 4 children were more likely to be overeaters and have obese mothers.

Conclusion The characteristics of residential environments may play a role in childhood weight status beyond social and early life factors. These characteristics may have differing effects within the population, so a 'one-size fits all' strategy for intervention may not be appropriate. 